\title{
High Dynamic Range Mixer in CMOS 0.18 um Technology for WLAN Direct Conversion Receiver
}

\author{
O. Nizhnik, R.K. Pokharel, H. Kanaya, and K. Yoshida \\ Graduate School of Information Sciences and Electrical Engineering \\ Kyushu University, Fukuoka 819-0395, Japan
}

\begin{abstract}
This paper describes simulation results of high dynamic range mixer, based on resistive ring topology. Mixer is designed using TSMC CMOS 0.18um technology. This mixer downconverts RF signals in the range 2-6 GHz to low IF using LO signals below corresponding RF frequencies and perform low-pass filtering with 3dB bandwidth $33 \mathrm{MHz}$. Unlike pure resistive ring topology, mixer has a positive power gain. Output 2 nd and 3 rd order intercept point for proposed mixer is $41 \mathrm{dBm}$ and $22 \mathrm{dBm}$, resulting in dynamic range over 60dB even for high-throughput transceivers. Mixer have an integrated $\mathrm{LO}$ drive with gain $6 \mathrm{~dB}$, so does not require significant LO power to operate. The mixer has DC power consumption $54 \mathrm{~mW}$ and can be used in the most demanding ultra-wideband OFDM radio and WLAN communication systems.
\end{abstract}

\section{INTRODUCTION}

In the modern wireless communication networks two standards are becoming increasingly popular. First is 802.15.3 High Rate WPAN, and second is 802.11 WLAN.

Both standards are using OFDM scheme to increase data throughput. But one of the main problems of OFDM system remained inter-channel interference. For WLAN desirable adjacent channel rejection is about $60 \mathrm{~dB}$, and for WPAN due to larger number of OFDM channels requirements are even more strict. ACPR requirement necessitate high-linearity solution for entire transceiver, including mixer. Linearity of common workhorse of RF transceivers, Gilbert cell mixer is not satisfactory in modern communication systems. Although in [1],[2],[3] was proposed some solutions to increase linearity of wideband Gilbert cell mixer, all of these solutions still have dynamic range around $30-40 \mathrm{~dB}$ and thus unsuitable for WPAN communication or even for broadband WLAN communications. Now industry chiefly needs wideband, high-linearity mixer. Wide known resistive ring mixer meets both requirements, but have significant drawbacks - conversion loss and high LO power 。

In the proposed design both problems are alleviated, but DC power consumption is slightly increased. Also using analog postdistortion technique, linearity of mixer is further boosted by approximately $10 \mathrm{~dB}$, and first time low-pass filter implementation is based on skin-effect induced loss. This is the first time a filter with cutoff frequency as low $33 \mathrm{MHz}$ implemented on-chip without using noisy active elements, resulting in superior noise figure $11.5 \mathrm{~dB}$ at IF $10 \mathrm{MHz}$. Superior noise performance makes proposed mixer suitable for direct conversion receivers.

\section{COMPOSITION OF MIXER}

The mixer comprises of the following key components: RF matching circuit, LO differential power driver, resistive ring mixer core, postdistortion amplifier, low-pass filter as shown in Fig. 1.

Postdistortion amplifier is implemented using matched pair of cascode amplifiers. Low-pass filter consists of 2 identical LC pisections. Cutoff of the low-pass filter is determined by skin effect equation so ultra-thick top metal of filter inductors (see Fig. 2) is necessary to reduce cutoff frequency of the filter.

Postdistortion amplifier make use of different sign of quadratic nonlinearity at weak inversion and strong inversion for deepsubmicron MOSFET transistors. Transistors of resistive ring is biased into strong inversion region, while transistors of postdistortion amplifier biased into weak inversion region, thus nullification of quadratic distortion is possible.

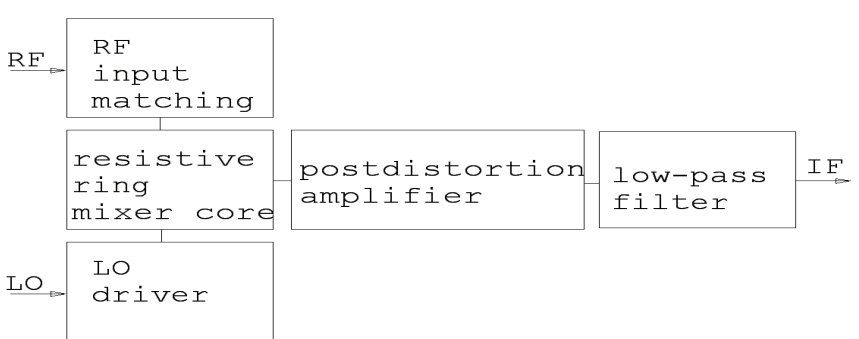

Figure 1. Block diagram of proposed high dynamic range mixer

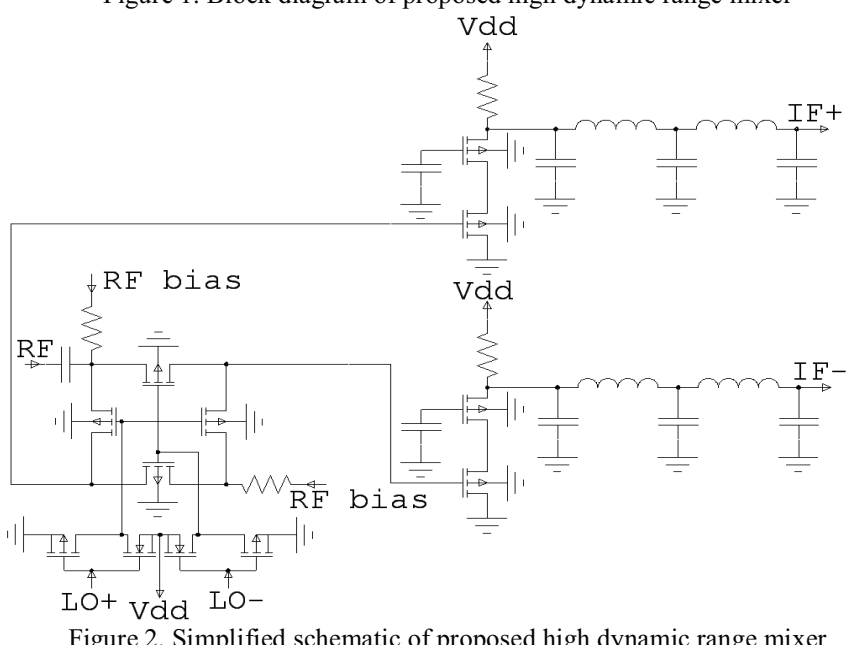




\section{SIMULATION RESULTS}

Because output of mixer is usually high-impedance, effort should be made for large and flat voltage gain. But proposed mixer was optimized not for gain flatness, but for minimal noise figure. Because mixer is most noisy at high frequency, gain optimized to be maximal close to maximal operating frequency (see Fig. 3). For same reason the size of resistive ring transistors was increased compared with [4] 4-fold. Resulting degradation of bandwidth allowed dramatic reduction of in-band noise figure.

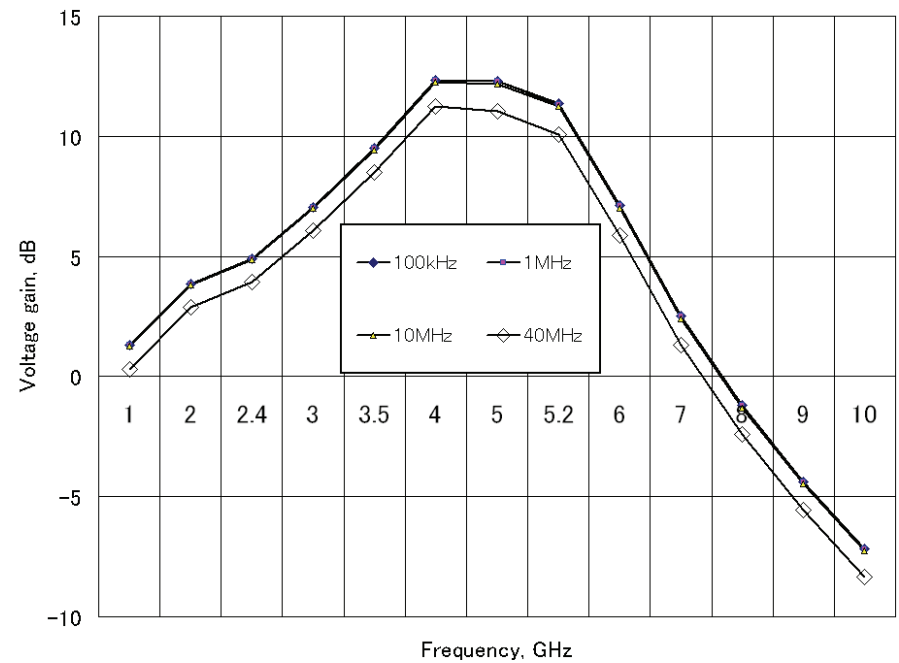

(see Fig.4)

Figure 3. Voltage gain of high dynamic range mixer, $d B$ (simulated post-layout)

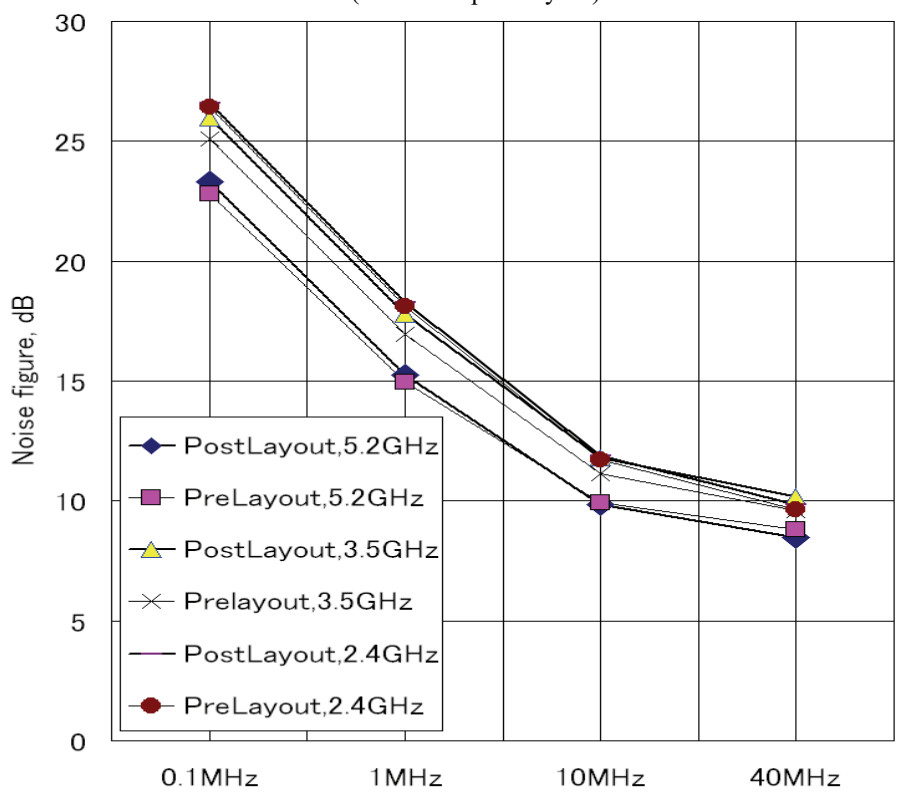

IF frequency

Figure 4. Noise figure of high dynamic range mixer, dB (simulated)
Thermal noise floor of mixer is $8 \mathrm{~dB}$ and flicker noise corner frequency estimated to be $6 \mathrm{MHz}$. The $70 \%$ of flicker noise attributed to postdistortion amplifiers and $25 \%$ to resistive ring mixer core. Other 5\% comes from local oscillator driver.

Thermal noise is dominated by the resistive ring core, leaving only a small contribution to RF matching circuit and LO driver. Along with preceding LNA, having $2.5 \mathrm{~dB}$ noise figure and $15 \mathrm{~dB}$ gain, RF front-end noise figure can be kept below $4 \mathrm{~dB}$ at IF $10 \mathrm{MHz}$ and above, making mixer very attractive for direct conversion architecture. If the minimal IF frequency is set to $1 \mathrm{MHz}$, overall RF front-end noise figure still can be below $5.5 \mathrm{~dB}$

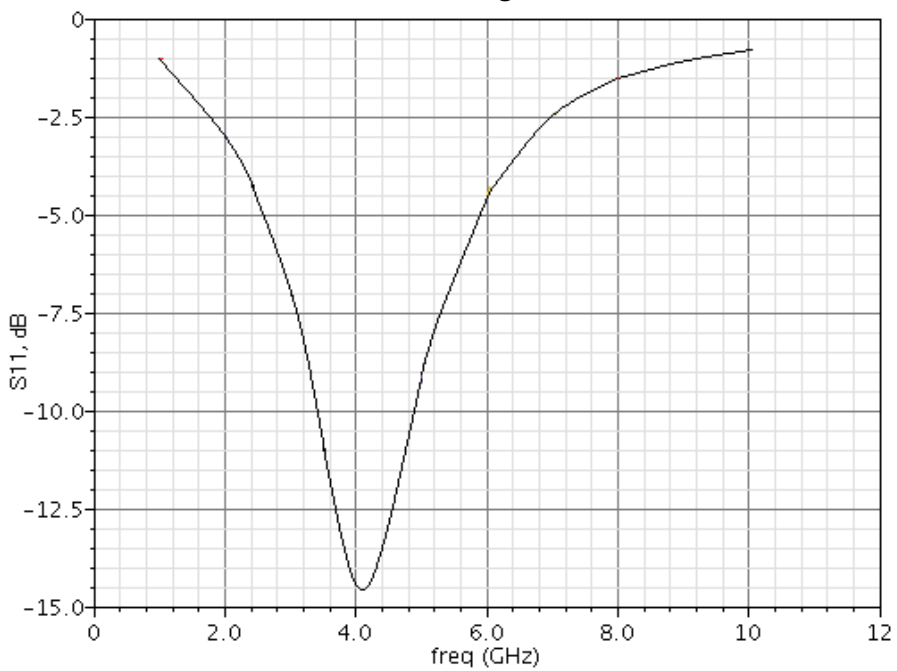

Figure 5. Input return loss of high dynamic range mixer, $\mathrm{dB}$ (simulated post-layout)

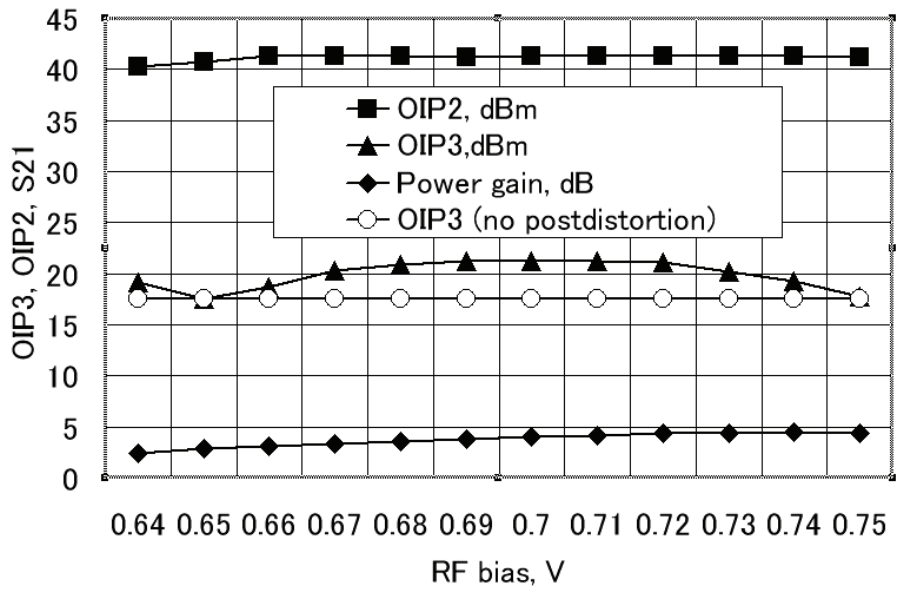

Figure 6.Power gain and intercept points of high dynamic range mixer (simulated post-layout)

The input matching (see Fig. 5) for proposed mixer is narrowband. Wideband matching was considered impractical, because unlike LNA connected to strongly reflecting antenna, mixer is connected to weakly reflecting output of LNA. So large 
input reflection do not hamper stability and can be tolerated as long as noise matching is close to optimal. Simultaneous gain and noise matching is not implemented mixer design not to increase passive components count.

On the plot of worst-case intercept points (see Fig. 6) clearly visible peak of the OIP3 at RF bias around $0.7 \mathrm{~V}$. Amplitude of peak is $5 \mathrm{~dB}$, corresponding to $10 \mathrm{~dB}$ linearization of resistive ring mixer. This confirmed by large figure-of-merit characterizing linearity.

$$
F O M=\frac{O I P 3}{P_{D C}+P_{A C}}=\frac{158 m \mathrm{~W}}{67 \mathrm{~mW}}=2.4
$$

Linearization of mixer is caused approximately $1 \mathrm{~dB}$ gain loss and $1 \mathrm{~dB}$ noise figure increase, but it is compensated by $10 \mathrm{~dB}$ increase of dynamic range. Taking into account thermal noise and distortion of following sample-and-hold circuit, optimal input power for proposed mixer is $-20 \mathrm{dBm}$. At this input power dynamic range is limited by $2^{\text {nd }}$ order distortion, with $3^{\text {rd }}$ order distortion making significant contribution only if input RF power more then $-6 \mathrm{dBm}$.

In [4], was proposed to use output buffer, but authors did not used output buffer as means of linearization. Instead buffer was used only to compensate large conversion loss of small transistors in ultra-wideband resistive ring.

In [5] design of mixer had good linearity, but limited by the large leakage of LO signal. So combining low-pass filter and postdistortion amplifier addressed both major issues in designs [4] and [5].

In [6] was proposed to use alternative approach to linearization, i.e. bootstrapped switch. Although performance of bootstrapped linearizer is close to postdistortion linearizer, the disadvantage was the huge bandwidth reduction, typical for bootstrapped switches.

\begin{tabular}{|c|l|l|l|l|}
\hline \multicolumn{5}{|c|}{ Table 1. Comparison of wideband mixers } \\
\hline Parameter & Work [4] & Work [5] & Work[6] & This work \\
\hline Voltage gain & $-7.5 \mathrm{~dB}$ & $-5.8 \mathrm{~dB}$ & $>8 \mathrm{~dB}$ & $>+3 \mathrm{~dB}$ \\
\hline Bandwidth & $1-11 \mathrm{GHz}$ & $0.9-1.8 \mathrm{GHz}$ & $2.3-2.6 \mathrm{GHz}$ & $2-6 \mathrm{GHz}$ \\
\hline $\begin{array}{c}\text { Noise figure } \\
@ \text { IF }\end{array}$ & $\begin{array}{l}7.5 \mathrm{~dB} \\
@ 10 \mathrm{MHz}\end{array}$ & $5.8 \mathrm{~dB}$ & $\begin{array}{l}11 \mathrm{~dB} \\
@ 7 \mathrm{MHz}\end{array}$ & $\begin{array}{l}11.5 \mathrm{db} \\
@ 10 \mathrm{MHz}\end{array}$ \\
\hline LPF cutoff & - & - & - & $50 \mathrm{MHz}$ \\
\hline DC power & $3 \mathrm{~mW}$ & 0 & 0 & $54 \mathrm{~mW}$ \\
\hline LO power & $8 \mathrm{~mW}$ & $25 \mathrm{~mW}$ & $\approx 25 \mathrm{~mW}$ & $13 \mathrm{~mW}$ \\
\hline OIP3 & $+4 \mathrm{dBm}$ & $+13.8 \mathrm{dBm}$ & $0 \mathrm{dBm}$ & $+22 \mathrm{dBm}$ \\
\hline OIP2 & not known & not known & not known & $+42 \mathrm{dBm}$ \\
\hline Linearity FOM & 0.2 & 1 & 0.04 & 2.4 \\
\hline Dynamic range & $48 \mathrm{~dB}(\mathrm{IM} 3)$ & $43 \mathrm{~dB}(\mathrm{LO}$ & $\begin{array}{l}40 \mathrm{~dB} \\
(\mathrm{IM} 3)\end{array}$ & $>58 \mathrm{~dB}(\mathrm{IM} 2)$ \\
\hline
\end{tabular}

Table 1 illustrates proof what proposed mixer composition is superior in signal fidelity over all competing mixers. Signal dynamic range was calculated assuming RF input power $-20 \mathrm{dBm}$.
Table 2. Technical parameters of proposed high dynamic range mixer

\begin{tabular}{|c|c|}
\hline Parameter & Value \\
\hline Input return loss & $<-10 \mathrm{~dB}$ \\
\hline Chip area (2 sizes of LPF possible) & $2 \mathrm{~mm}^{2}$ or $3 \mathrm{~mm}^{2}$ \\
\hline 1dB IF output compression point & $+1 \mathrm{dBm}$ \\
\hline Supply voltages & $1.8 \mathrm{~V}$ power and $0.7 \mathrm{~V}$ bias \\
\hline IF output maximal voltage swing & $0.8 \mathrm{~V}$ p-p \\
\hline
\end{tabular}

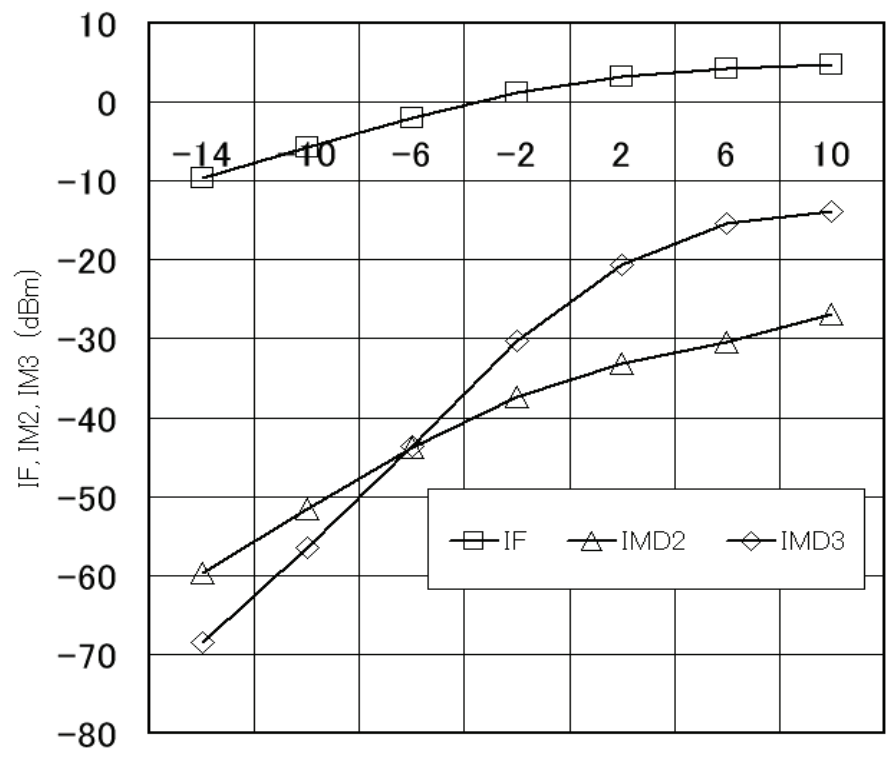

RF input power, dBm

Figure 7. IF signal and main contaminating signals at the output of high dynamic range mixer

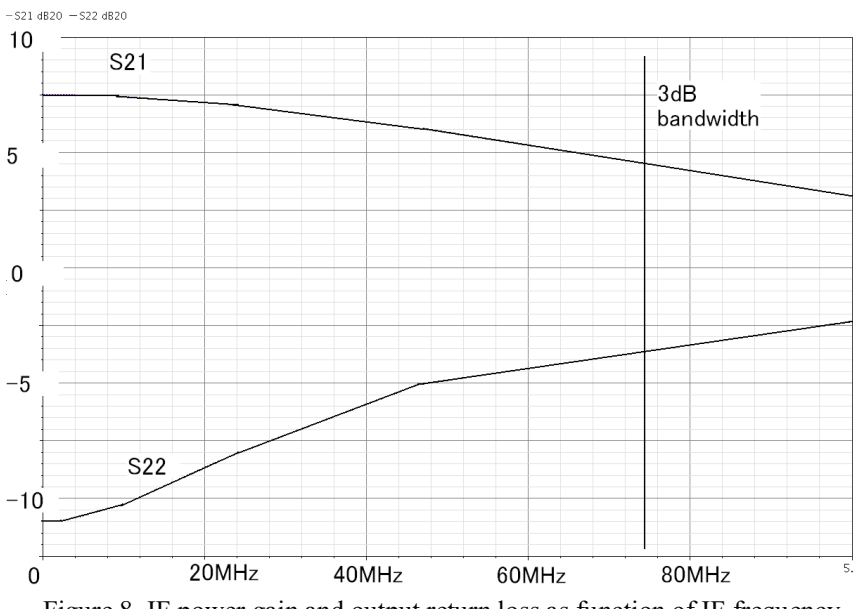

Figure 8. IF power gain and output return loss as function of IF frequency for $66 \mathrm{MHz} \mathrm{LPF}$.

Mixer was designed and sent to fabrication in 4 variants - with $33 \mathrm{MHz}$ or $66 \mathrm{MHz}$ low-pass filters, and also with compact LO drive, requiring $13 \mathrm{~mW}$ input power or high-gain LO drive with digital phase splitter. Low-pass filter cutoff is shown on Fig.8. 


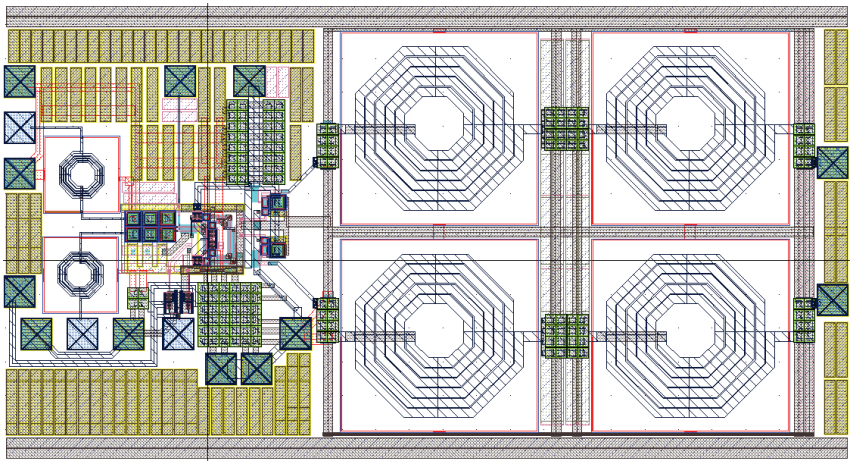

Figure 9. Layout of wideband mixer with integrated LO phase splitter and $66 \mathrm{MHz}$ low-pass filters. Total chip area $3 \mathrm{~mm} 2$

Although high-gain LO driver improve integration of mixer solution, with 0.18 um Si CMOS technology it expected to limit maximal operating frequency to $4 \mathrm{GHz}$, because classical digital drivers fail to propagate signal at frequencies above $4 \mathrm{GHz}$, if 0.18 um TSMC CMOS technology is used. So LO driver become the bandwidth-limiting factor for proposed mixer.

$1 \mathrm{~dB}$ output compression point for mixer lies at $+1 \mathrm{dBm}$ (see Fig. 7 ), what confirms reliability of $-20 \mathrm{dBm}$ optimal input power point for the mixer. But the mixer can also work at RF input power as high as $-6 \mathrm{dBm}$ and still have $40 \mathrm{~dB}$ dynamic range.

\section{CONCLUSION}

A 0.18um CMOS mixer, based on resistive ring topology was designed, simulated and sent for fabrication. The mixer has a large bandwidth covering from $2 \mathrm{GHz}$ to $6 \mathrm{GHz}$ and suitable for direct conversion receivers. The chip area, including $66 \mathrm{MHz}$ lowpass filter, is $3 \mathrm{~mm}^{2}$ (see Fig. 9) Proposed mixer have an OIP3 more then $+22 \mathrm{dBm}$ and OIP2 more then $+41 \mathrm{dBm}$. Unlike more conventional mixers, dynamic range limited by IM2 instead IM3. For IEEE 802.15.3 systems (WPAN) distortion is equal to thermal in-band noise leaving no reasons to reduce IM2 and IM3 further. Proposed mixer is suitable for WLAN and WPAN wideband communication systems with strict requirements for dynamic range. Dynamic range of mixer reaches $60 \mathrm{~dB}$.

\section{ACKNOWLEDGEMETS}

This work was partly supported by a grant of Knowledge Cluster Initiative implemented by Ministry of Education, Culture, Sports, Science and Technology (MEXT). This work was also partly supported by VLSI Design and Education Center (VDEC), the University of Tokyo in collaboration with CADENCE Corporation and Agilent Corporation.

\section{REFERENCES}

[1] Ming-Da Tsai and Huei Wang, "A 0.3-25-GHz Ultra-Wideband Mixer Using Commercial 0.18-um CMOS Technology”, IEEE Microwave And Wireless Components Letters, vol. 14, No. 11, pp.522-524, November 2004. [2] Fong-Cheng Chang, Ping-Cheng Huang, Shih-Fong Chao and Huei Wang, "A Low Power Folded Mixer for UWB System Applications in 0.18-um CMOS Technology", IEEE Microwave And Wireless Components Letters, vol. 17, No. 5,pp. 367 - 369, May 2007.

[3] Motieifar, A., Pour, Z.A., Bridges, G., Shafai, C. and Shafai, L., "An Ultra Wideband (UWB) Mixer with 0.18UM RF CMOS Technology”, Electrical and Computer Engineering, Canadian Conference on May 2006 Page(s):697700 .

[4] Tienyu Chang and Jenshan Lin, "1-11 GHz Ultra-Wideband Resistive Ring Mixer in 0.18-um CMOS technology", Radio Frequency Integrated Circuits (RFIC) Symposium, 2006 IEEE.

[5] Gould, P., Zelley, C. and Lin, J.,"A CMOS resistive ring mixer MMICs for GSM 900 and DCS 1800 base station applications", Microwave Symposium Digest., 2000 IEEE MTT-S International Volume 1, pp. 521 - 524, 11-16 June 2000 .

[6] Tillman F. and Sjoland, H., "A bootstrapping technique to improve the linearity of CMOS passive mixers", VLSI Circuits, 2003. Digest of Technical Papers, pp.221-222, 12-14 June 2003. 